\title{
微结构可控材料的制备及其在生物医学的 应用
}

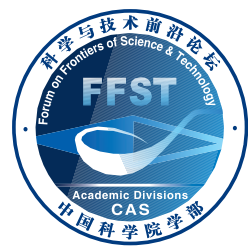

\author{
丁海波, 刘柯良, 卫孟萧, 郭一君, 顾洪成, 顾忠泽 ${ }^{*}$
}

东南大学生物科学与医学工程学院, 生物电子学国家重点实验室, 南京 210096

*通讯作者, E-mail: gu@seu.edu.cn

收稿日期: 2021-03-31; 接受日期: 2021-05-14; 网络版发表日期: 2021-05-18

国家重点研发计划(编号: 2017YFA0700500)和国家自然科学基金(编号: 21635001, 52033002, 52003051, 21902024)资助项目

摘要微结构可控材料是一类利用微观结构调控整体性能的新材料. 在不改变材料本身物理化学性质的条件 下，通过控制微结构组成单元的尺寸、几何构型、排列方式提升宏观材料的力学、热学、表界面等性质. 在微结 构可控材料的研究中, 性能调控的关键在于跨尺度分级结构的精准构筑. 本文从制备工艺出发概述了微结构可控 材料的最新研究进展, 着重介绍了聚合物、金属及无机材料的跨尺度微纳加工方法对于整体性能的提升作用, 并 总结了微结构可控材料在生物医学领域中低密度材料、微液滴操控、微型机器人等方面的应用, 展望了其未来 的发展方向.

关键词微结构可控材料, 跨尺度, 增材制造, 生物医学

\section{1 引言}

微结构可控材料是指利用微观结构设计调控整体 性能的一类人工材料 ${ }^{[1]}$. 对于这类材料而言, 微结构组 成单元的尺寸、几何构型、排列方式等结构参数能够 与本体材料的物理化学性质协同增效, 实现宏观性质 (如力学、热学、界面等)的精准调控 ${ }^{[2,3]}$. 随着纳米技 术研究的深入，微纳尺度下传统材料的新性能被不断 发现. 通过组合具有尺寸效应的微纳结构、周期性结 构、分级结构以及响应性结构，微结构可控材料在微 机械、微电子以及智能传感等方面具有广泛的应用 前景.

一个典型的微结构可控材料是基于金属微晶格的
低密度超强材料 ${ }^{[4]}$. 如图1所示, 该微晶格材料由镍中 空管以 60 度倾角组成, 其中镍中空管的长度为 1 至 4 毫 米，管道直径 100 至 500 微米，管道厚度为 100 至 500 纳 米. 这样的结构设计不仅将整体密度降低到每立方厘 米 0.9 毫克, 同时仍然具有良好的机械性能, 能够在压 缩 $50 \%$ 后恢复原貌. 因此, 这种低密度超强材料成为未 来航天器件的备选材料.

对于微结构可控材料而言, 其面临的挑战在于成 型方法, 不仅需要构筑覆盖厘米-毫米-微米-纳米的跨 尺度分级结构, 还要能够精准控制指定聚合物、金属 或无机材料的分布. 传统的机械加工、模板拓印、化 学组装等技术难以应对这样的严苛要求. 直到增材制 造技术的兴起使得特征尺寸小、复杂度高的结构成型

引用格式: Ding H, Liu K, Wei M, Guo Y, Gu H, Gu Z. Fabrication of controlled microstructural architectures and application in biomedical engineering. Sci Sin Chim, 2021, 51: 1501-1510, doi: 10.1360/SSC-2021-0073 


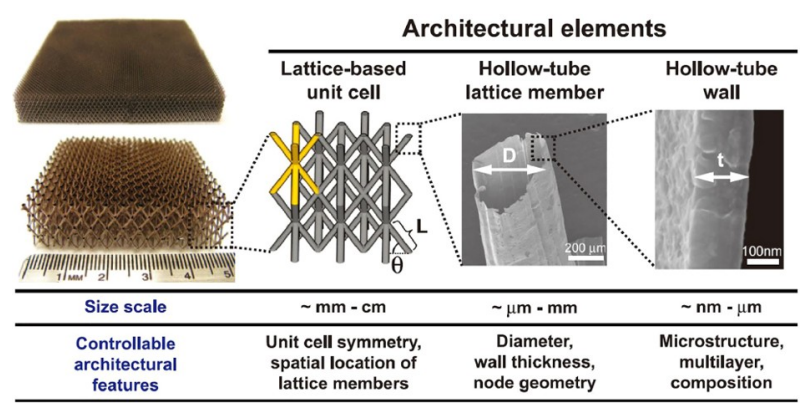

图 1 超轻金属微晶格材料实物及微结构表征图 ${ }^{[4]}$ (网络版 彩图)

Figure 1 Images of the ultralight metallic microlattices and the detailed microstructures [4] (color online).

成为可能, 有力地推动了微结构可控材料的科学研究 和实际应用. 本文中，我们归纳了微结构可控材料制 备中所涉及的跨尺度微纳加工方法，通过阐述不同工 艺的工作原理总结出各自的性能特点和适用目标，最 后介绍了微结构可控材料在生物医学领域的具体应用 和前景展望.

\section{2 微结构可控材料的制备}

针对微纳结构的制备，实验室可选用的增材制造 技术主要包括熔融沉积、喷墨打印、直接墨水书写、 立体光刻、双光子聚合等 ${ }^{[5,6]}$. 其中，熔融沉积、喷墨 打印、直接墨水书写等技术将融化后的聚合物、金属 或液态墨水等材料通过喷头挤出，在指定位置完成固 化成型. 其打印精度受限于喷头口径，难以达到微米 以下; 成型结构依靠位移台的相对移动，面对三维悬 梁结构时需要额外的支架辅助. 上述两点不足使得这 类基于墨水成型的增材制造技术无法满足微结构可控 材料中复杂结构的制备.

与之相比，基于光聚合的立体光刻、双光子聚合 等增材制造技术具有加工精度高、成型质量好的优 点, 可以为不同材料的目标结构提供基本支架. 当目标 材料为聚合物时，通过光聚合原理直接制备相应的聚 合物支架; 当目标材料为金属时，以聚合物支架为模 板实施金属沉积并去除聚合物模板; 当目标材料为碳 材料时, 以聚合物支架为基础实施高温热处理. 因此, 基于光聚合反应的增材制造技术成为微结构可控材料 的首要选择. 制备的聚合物支架保证了人工设计跨尺 度分级结构的成型，后续金属沉积和热处理使得整体
性能产生进一步的提升.

\section{1 基于光聚合反应的增材制造}

光聚合反应是指曝光产生的自由基分子引起聚合 物单体发生的聚合反应. 根据自由基产生过程中, 光敏 物质吸收光子数的多少, 可以分为单光子聚合和多光 子聚合 ${ }^{[7,8]}$. 单光子吸收所需的能量较低, 在紫外光源 激发下, 曝光区域均会生成大量自由基而发生聚合. 而 多光子吸收需要高强激光的激发, 通常只能发生在超 快激光的聚焦焦点，产生的有效聚合区域具有明显的 空间限域效应. 聚合反应原理的差异直接决定了实际 加工过程和加工效果. 现有文献报道中主要包括基于 单光子聚合的自导光聚合、面投影微立体光刻以及基 于双光子聚合的三维激光直写.

\subsection{1 自导光聚合}

对于单光子聚合反应，通常采用紫外光作为激发 光源，利用掩模版来控制曝光区域以加工不同形状的 二维目标. 为了实现三维结构的加工, 大阪大学的Kawata等人 ${ }^{[9]}$ 开发了一种自导光聚合技术用于周期性网 络的加工. 在实施过程中, 一方面利用掩模版控制二维 曝光图案, 另一方面通过激发光的传播实现曝光图案 在三维空间的延伸(图2). 由于固化后的聚合物材料与 尚未固化的光刻胶中间存在折射率差异，使得入射激 光能够保持初始入射角度，聚合反应能够随着光线传 播方向深入到光刻胶内部, 形成网络状固化结构. 图1 中超轻金属微晶格材料的聚合物支架正是使用该方法 制备. 但是, 由于光线传播方向无法改变, 这种加工方 法仅适用于贯穿网络的快速制备，无法满足复杂构型 的结构加工.

\subsection{2 面投影微立体光刻}

为了解决紫外聚合反应加工复杂三维支架的问 题, 面投影微立体光刻应运而生 ${ }^{[10]}$. 如图3所示, 其具体 操作步骤包括: (1) 将加工目标的数字模型按固定厚度 分层; (2) 将每一层模型切片对应的入射光投射到光刻 胶界面; (3) 利用位移台控制分层的叠加组装; (4) 利用 显影液去除未聚合的光刻胶, 完成干燥成型. 与自导光 聚合相比，面投影微立体光刻在三维成型能力和加工 精度上得到明显提升. 由于该方法不依赖于实体的光 掩模版来控制加工形状, 而是通过数字微镜器件、微 


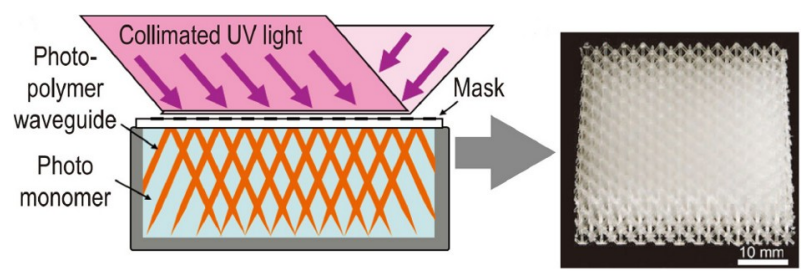

图 2 自导光聚合示意图及微结构实物图 ${ }^{[1]}$ (网络版彩图)

Figure 2 Scheme of the self-propagating polymerization and images of the fabricated polymer microstructure [1] (color online).

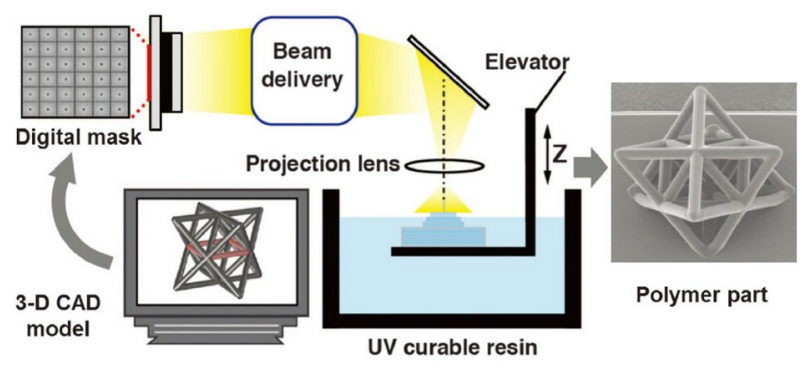

图 3 面投影微立体光刻示意图及微结构实物图 ${ }^{[10]}$ (网络版 彩图)

Figure 3 Scheme of the projection micro-stereolithography and images of the fabricated polymer microstructure [10] (color online).

透镜阵列或空间光调制器等设备直接调制入射光的形 状, 增加了图案变化的灵活度. 同时, 在光路中可以通 过透镜聚焦控制实际投射图案的大小，能够满足更加 复杂、更加精细的聚合加工.

2006年，加州大学洛杉矶分校Zhang等人 ${ }^{[11]}$ 确立 了以数字微镜器件为动态掩膜的面投影微立体光刻技 术. 为了提高加工效率, 美国Carbon3D公司 ${ }^{[12]}$ 开发出 连续液体界面制造技术，通过透氧膜调节光刻胶聚合 界面处氧气浓度，达到抑制自由基聚合的目的．基于 该技术，面投影微立体光刻的加工速度提升了1000倍. 为了提高打印体积, 美国西北大学Mirkin团队 ${ }^{[13]}$ 在光 刻胶聚合界面设置一层流动的氟化油来去除光固化过 程中热效应累积, 防止新生成层与基座的粘连. 使用该 方法每小时可加工 $430 \mathrm{~mm}^{3}$ 的目标，3小时可加工出 $0.3 \times 0.3 \times 1.2 \mathrm{~m}^{3}$ 的晶格结构. 为了提高加工精度, 德国 勃兰登堡应用科学大学Regehly团队 ${ }^{[14]}$ 基于双色聚合 原理控制聚合反应发生在两束激光的交汇处，一方面 利用垂直方向光投影调节聚合形状，另一方面利用侧 向入射光控制聚合的厚度及位置. 由于采用光片聚焦 技术控制侧向入射光，因此该方法下加工精度提高至 10 微米级别.

\subsection{3 双光子聚合}

受紫外聚合原理的限制，面投影微立体光刻在实 际加工中也受到两个限制：（1）加工精度上难以突破 亚微米级; (2) 加工模式必须依赖于层层堆叠. 对于微 尺度可控结构而言, 这两方面的不足造成一些精细、 复杂结构难以实现.

双光子聚合的空间限域特性正好弥补这两方面的 不足. 由于聚合反应需要达到一定的能量阈值, 因此双 光子聚合中有效聚合结构的尺寸可以突破衍射极限; 另一方面，空间限域效应支持双光子聚合反应在三维 空间直写加工, 为复杂微结构的构建提供了可能 ${ }^{[15,16]}$. 2013年, 加州理工大学的Greer团队 ${ }^{[17]}$ 首次将双光子聚 合技术引入微结构可控材料的制备，其制备的聚合物 结构整体尺寸达到 100 微米以上, 最小支架直径达到 250 纳米.

为了提升微结构可控材料的性能, 纳米光子学领 域的最新进展被运用于双光子聚合, 以提升加工精度 和加工效率 ${ }^{[18]}$ (图4). 对于加工精度而言, 双光子聚合 可以通过调节激光能量或者曝光时间的方法控制有效 聚合区域的大小. 但这样的调节方式往往造成聚合得 到的结构聚合度低、机械强度差, 难以在显影后保存. 为了得到尺寸更加精细且聚合度高的结构, 受激发射 损耗技术被应用于双光子聚合中 ${ }^{[19,20]}$, 增加的抑制光 束可以在较强的激发光下进一步缩小有效聚合区域的 大小. 2013年，顾敏团队 ${ }^{[21]}$ 基于该原理实现了最小线 宽9纳米、最窄间距52纳米的世界记录.

另一方面，双光子聚合由于采用逐点扫描的成型

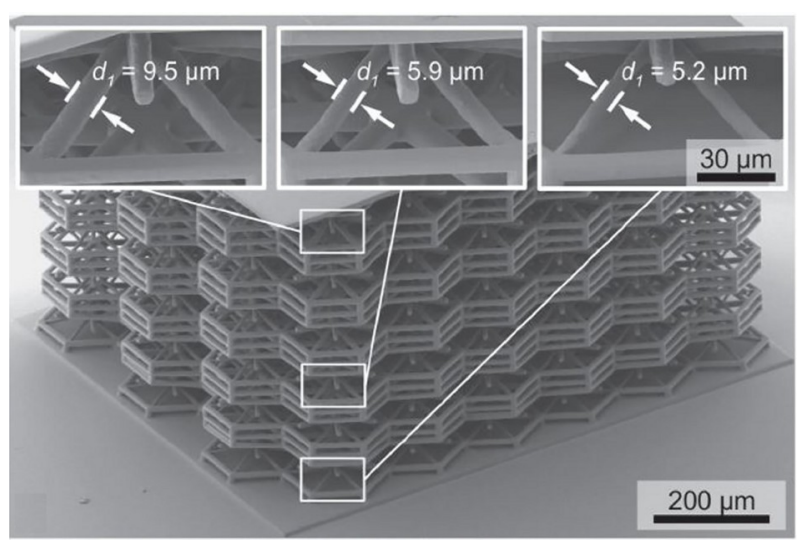

图 4 双光子聚合的微结构实物图 ${ }^{[18]}$ (网络版彩图)

Figure 4 Images of the polymer microstructure fabricated by twophoton polymerization [18] (color online). 
模式, 其加工效率远远低于面投影立体光刻技术. 亚微 米级的激光焦点导致加工精度与加工效率难以兼顾、 互为矛盾. 针对周期性结构的加工, 基于多焦点阵列的 并行激光直写技术被开发 ${ }^{[22,23]}$. 其中, 微透镜阵列和空 间光调制器保证了可编辑、高质量多焦点阵列的生 成. 2019年, 美国劳伦斯国家实验室Saha和中国香港大 学Shih-Chi Chen的团队 ${ }^{[24]}$ 结合时空压缩和多焦点阵列 技术，实现了 $165 \times 165$ 平方微米范围内最高 100 万像素 点的投影，单层厚度可达 1 微米以下，层间切换时间仅 20 毫秒. 此外, 利用波前调制技术调控飞秒激光, 使得 聚焦光场范围内光强分布按照目标形状形成投影，从 而突破点状高斯分布的限制，实现指定结构的单次曝 光加工, 达到提升加工效率的效果 ${ }^{[25,26]}$.

\subsection{4 光聚合加工方法总结}

表1总结了跨尺度分级结构制备过程中所常见的 三种光聚合加工方法. 通过对比可以总结出不同结构 目标最适合的加工方法：(1)面对特征尺寸在微米级以 上贯穿周期性结构时，自导光聚合方法可以满足快速 制备; (2)面对特征尺寸在微米级以上的三维构型时, 数字光投影可以提供兼顾精度与效率的加工; (3)面对 特征尺寸在微米级以下或复杂三维结构时，只能选择 双光子聚合加工.

\section{2 金属镀层}

金属、合金或金属氧化物材料对于提升微结构可 控材料的力学、电学、热学等性质具有重要作用. 常 用的加工方法是采用磁控溅射、原子层沉积、化学镀 等方法在制备的聚合物支架表面沉积相关材料，并通 过后续化学腐蚀或者热处理去除聚合物支架. 此外, 金属光还原技术的发展也推动了金属微结构的直写 加工.

\subsection{1 金属沉积}

常用金属镀膜等技术可以在真空或者惰性气体环 境下通过加热蒸发、磁控溅射等方法使靶材原子在目 标结构表面沉积. 这些方法在宏观尺度下能够实现金 属材料大面积快速成膜，但面对部分微结构可控材料 中的精细三维结构时，容易发生层厚不均匀的问题， 甚至出现加工死角 ${ }^{[27,28]}$.

针对上述缺陷，近些年兴起的原子层沉积技术通
过气相前驱体在基底材料上化学吸附形成沉积膜 (表 2). 该方法具有优异的自限制性, 每次反应只能沉积一 层原子, 有利于控制沉积膜的成份和厚度. 虽然该方法 反应时间长、加工成本高，但仍然成为高质量样品制 备的首选. 在微结构可控材料的研究中, 相关课题组 以制备的聚合物支架为模板，通过原子层沉积法在其 表面构筑均匀的氧化铝或氮化钛等陶瓷或合金镀层, 其厚度通过调控反应时间精准控制在纳米级别 ${ }^{[10,17,29]}$.

\subsection{2 化学镀}

化学镀是一种简便的材料表面金属处理技术．与 金属沉积相比，化学镀操作过程中需要将加工目标完 全浸入反应溶液中，借助合适的还原剂在非导体的表 面发生还原反应，从而将溶液中金属离子还原成金属 以形成致密金属层. 具体实施过程中, 可以在几分钟 内实现微米级厚度镍、铜等金属层的加工 ${ }^{[1,10,30]}$. 针对 微结构可控材料的制备, 该方法非常适合在复杂构型 的聚合物表面构建完整镀层，缺陷在于镀膜层厚尚不 能控制到纳米尺度.

\subsection{3 双光子还原}

除了以聚合物为模板间接制备金属结构外，光子 吸收过程中产生的自由基可以用于金属离子的还原， 尤其是双光子吸收效应更是为金属微纳结构的直写加 工提供了可能 ${ }^{[31,32]}$. 具体实施的系统与双光子聚合激

表 1 光聚合加工方法总结

Table 1 Summary of the photopolymerization methods

\begin{tabular}{cccc}
\hline & 自导光聚合 & 面投影立体光刻 & 双光子聚合 \\
\hline 加工方式 & 沿光线延伸 & 层层堆叠 & 逐点扫描 \\
是否需要模板? & 是 & 否 & 否 \\
加工精度 & 微米级别 & 微米级别 & 亚微米级别 \\
加工范围 & 厘达级 & 厘米级 & 级到 \\
设备成本 & 低 & 毫米级 \\
\hline
\end{tabular}

表 2 原子层沉积方法总结

Table 2 Summary of the atomic layer deposition methods

\begin{tabular}{ccccc}
\hline 镀层材料 & 前驱体1 & 前驱体2 & 反应温度 & 单层反应时间 \\
\hline $\mathrm{Al}_{2} \mathrm{O}_{3}{ }^{[10]}$ & $\mathrm{AlMe}_{3}$ & $\mathrm{H}_{2} \mathrm{O}$ & $125^{\circ} \mathrm{C}$ & $90 \mathrm{~s}$ \\
$\mathrm{Al}_{2} \mathrm{O}_{3}{ }^{[29]}$ & $\mathrm{C}_{3} \mathrm{H}_{9} \mathrm{Al}$ & $\mathrm{H}_{2} \mathrm{O}$ & $150^{\circ} \mathrm{C}$ & $40 \mathrm{~s}$ \\
$\mathrm{TiN}^{[17]}$ & $\mathrm{TiCl}_{4}$ & $\mathrm{~N}_{2} / \mathrm{H}_{2}$ & $140^{\circ} \mathrm{C}$ & $20 \mathrm{~s}$ \\
\hline
\end{tabular}


光直写系统相同，将飞秒激光通过聚焦物镜照射在含 有金属离子的溶液或聚合物预聚液，通过调节激光能 量和曝光时间控制还原出的金属结构. 当基本介质为 溶液时，可以通过混入表面活性剂控制金属颗粒成核 过程，防止生成过大的金属颗粒，达到提高加工分辨 率和结构表面平整度的效果. 已有报道中，该方法可 实现 $160 \mathrm{~nm}$ 线宽的银线结构 ${ }^{[33]}$.

不过, 受制于局部热效应, 双光子还原得到的金属 结构机械性能和导电性能比较差. 针对这一问题, 可以 用聚合物前聚体替代溶液作为基本介质，在飞秒光的 焦点处同时开展聚合和还原加工(图5)。该方法下，还 原出的金属得到聚合物网络的支撑，其机械性能明显 增强. 2016年, 德国卡尔斯鲁厄理工学院Christopher Barner-Kowollik团队 ${ }^{[34]}$ 以Irgacure 2959 为光引发剂, 氯金酸为金属前驱体，丙烯酸修饰的聚乙二醇为聚合 物前聚体配置了新型水溶性光刻胶. 在加工过程中, 通过聚焦波长 $700 \mathrm{~nm}$ 的飞秒激光，同时实施聚合和还 原加工, 得到聚合物-金属混合材料. 通过后续热处理 $\left(200^{\circ} \mathrm{C}, 10 \mathrm{~min}\right)$ 融化混合体系中的金颗粒, 进一步增强 金属颗粒的连接，使得制备结构的导电性能达到块状 金的水平.

\section{3 热处理}

为了得到具有跨尺度三维晶格结构的陶瓷或碳材 料, 可以光聚合加工制备相应的基本模板, 通过后续热 处理完成目标结构和材料的制备.

2016年，美国HRL实验室Schaedler团队 ${ }^{[35]}$ 报道了 一类基于碳氧化硅陶瓷的晶格材料(图6). 在制备过程 中，他们首先通过自导光聚合或立体光刻技术制备聚 合物网格，随后利用紫外光实现后续固化，最后通过 $1000^{\circ} \mathrm{C}$ 的高温处理完成热解. 在热解过程中, 聚合物 结构损失 $42 \%$ 的质量, 并发生 $30 \%$ 的线性收缩. 与相同 密度的陶瓷泡沫相比，经过人工设计与制造得到的具 有规则结构的碳氧化硅晶格陶瓷的力学强度显著增强.

同年, 德国卡尔斯鲁厄理工学院的Bauer团队 ${ }^{[36]}$ 则 报道了一类碳纳米晶格材料. 基于双光子聚合制备的 聚合物晶格在 $900^{\circ} \mathrm{C}$ 的高温热解后完成碳化. 整体结 构发生 $80 \%$ 的均匀收缩, 支架直径缩小到直接加工难 以达到的 $200 \mathrm{~nm}$ 以下, 单个晶格周期降低到 1 微米. 在 碳化过程中, 不仅材料本身发生转变, 其特征尺寸也达 到直接加工无法实现的尺度. 使得支架结构的强度提

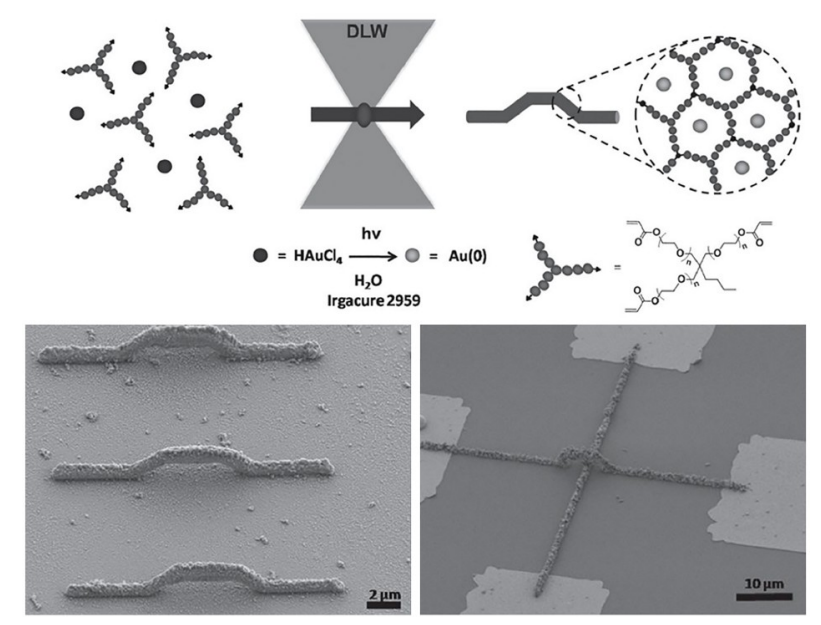

图 5 在聚合物基质中还原金属离子的示意图及制备的样 品 $^{[34]}$ (网络版彩图)

Figure 5 Scheme of photoreduction of metal ions in a polymeric matrix and an example of the fabricated microarches [14] (color online).
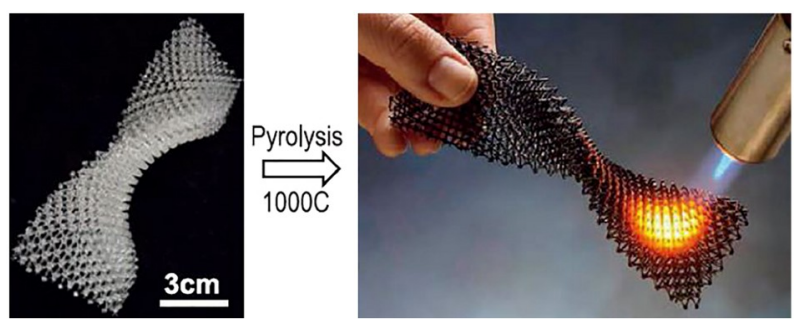

图 6 热解制备的碳氧化硅陶瓷晶格材料样品图 ${ }^{[35]}$ (网络版 彩图)

Figure 6 Images of the silicon oxycarbide microlattice fabricated by the pyrolysis of 3D-printed polymeric microlattices [35] (color online).

升至 $3 \mathrm{GPa}$ ，达到玻璃碳材料的强度理论值. 基于该方 法制备的蜂巢结构可以在 $0.6 \mathrm{~g} / \mathrm{cm}^{3}$ 的低密度条件下具 备高达 $1.2 \mathrm{GPa}$ 的压缩强度.

\section{3 微结构可控材料的生物医学应用}

随着加工工艺的发展, 微结构可控材料在力学、 表界面和应激形变方面的性能被发现和优化, 并在细 胞外环境构建、扫描探针成像、微液滴操作、微型机 器人等方面得到应用. 通过生物医学应用, 不仅可以为 微结构可控材料在其他方面的应用提供示范, 还能够 为加工方法与结构设计的提升提供用户反馈.

\section{1 可控力学材料}

可控力学是微结构可控材料发展的初衷. 美国加 
州理工大学的Greer、美国劳伦斯国家实验室的Spadaccini和麻省理工学院的Fang等团队 ${ }^{[37 \sim 41]}$ 围绕降低整 体密度、提升机械性能的目的，不断优化微晶格的结 构设计与加工方案，实现了弹性模量、剪切模量、泊 松比等力学参数的可控调节.

这种基于微晶格结构材料为生物相容性材料的力 学性质调节提供了解决方案. 为了构建与体内高度类 似的培养环境，细胞体外培养实验中不仅需要使用生 物相容性良好的材料, 还需要进一步控制材料的硬度 以保证细胞粘附与生长 ${ }^{[2,43]}$. 目前普遍的化学修饰、 纳米材料掺杂等方法在改变聚合物材料力学性质的同 时, 也引入了不可控的有毒物质. 而微晶格方案通过改 变晶格类型、晶格大小及周期性层数等参数，不仅可 以调控生物相容性材料的整体力学性能，还可以调控 空间上的力学性质差异分布，对于构建高仿真细胞外 基质、开展细胞诱导生长极具价值.

此外，这类微晶格材料还可以进一步用于微结构 的能量吸收与结构保护 ${ }^{[18,44]}$. 作为表征生物样品表面 形貌的重要工具, 扫描探针成像通过扫描探针与目标 样品的交互反馈, 描绘目标样品的三维轮廓. 然而, 柔 软的生物样品与坚硬的扫描探针之间存在模量失配的 问题，导致探针进针-退针过程中反馈滞后，且多次扫 描容易造成样品损坏. 针对这一问题, 本团队发展了一 种基于聚合物微晶格的扫描探针方案(图7) ${ }^{[45]}$. 在探针 设计过程中, 选用金刚石结构作为微晶格基本单元, 通 过控制晶格大小和整体层数调节探针弹性模量. 在探 针与目标材料接触过程中，探针将自身形变存储部分 运动动能, 加速系统能量衰减耗散，促使探针快速减 速至稳定接触状态，提高了探针力学反馈的灵敏度, 防止了过度接触造成的结果失真和样品损坏. 为了使 得探针与生物样品更加匹配，使用反应离子刻蚀进一 步缩小微晶格支架的直径至 $105 \mathrm{~nm}$ ，探针顶点直径 $17 \mathrm{~nm}$ ，进一步降低探针的弹性系数至 $0.12 \mathrm{~N} / \mathrm{m}$. 该探 针面对希瓦氏菌MR-1(弹性系数 $0.02 \sim 0.05 \mathrm{~N} / \mathrm{m}$ ) 时展 现出良好的灵敏度和稳定性，验证了微晶格探针方案 的有效性(图7e).

\section{2 可控浸润材料}

作为材料的基本性质之一，表界面的浸润性对于 液体流动、液滴捕获、油水分离等具有重要意义．在 生物医学研究中，调控材料的浸润性有利于保证微流
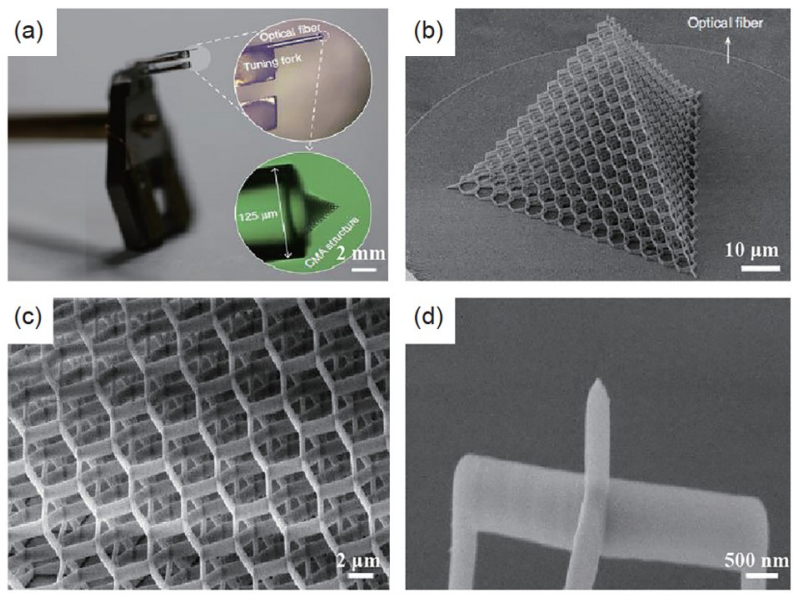

(d)

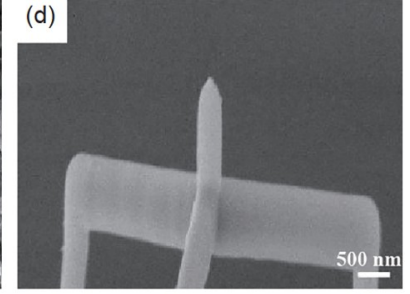

(e)
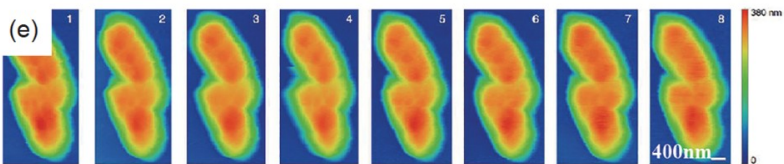

图 7 微晶格扫描探针 ${ }^{[45]}$. (a) 实物图; (b d ) 微晶格扫描电 镜图; (e) 希瓦氏菌MR-1的扫描成像图 (网络版彩图)

Figure 7 (a) Images of the 3D-printed cellular tips for atomic force microscopy; (b-d) Scanning electron microscopy (SEM) images of the microlattice structures. (e) Height profiles of Shewanella oneidensis MR-1 bacterial cells after repetitive scans [45] (color online).

道内的液体输运，生成高通量分析所需的微液滴阵列 以及混合溶液的成分分离. 但面对微流控芯片时，传 统化学修饰方案难以在微小空间内实现局部改性，以 改变指定位置的表面浸润性.

为此，本团队在跳虫皮肤表面的天然结构启发下， 设计了一种具有可控浸润性能的凹腔结构阵列用于液 滴操控 ${ }^{[46-48]}$. 该聚合物结构可以通过双光子聚合直接 制备, 无需额外的化学修饰. 受边缘效应的作用, 人造 凹腔结构阵列的临界角受结构本身几何构型与阵列中 单元排列的调控. 其中, 三重凹腔单元阵列比双重凹腔 结构阵列具有更大的临界角，不仅表现出超疏水效果， 对于低表面张力的有机试剂也表现出明显的排斥效 果 ${ }^{[46]}$. 而双重凹腔结构阵列表现出更加灵活的调控性 能，可以通过控制微结构单元的中心距实现液滴形貌 的调控 ${ }^{[47]}$. 因此，利用三重凹腔结构的超疏水、超疏 油性能可以构建约束液体输运的开放式流体通道; 而 在指定位置设置双重凹腔结构阵列，则可以达到锁定 液滴位置、调控液滴形貌的效果(图8).

\section{3 可控形变材料}

面对活动目标追踪、监测、去除等需求，具有远 

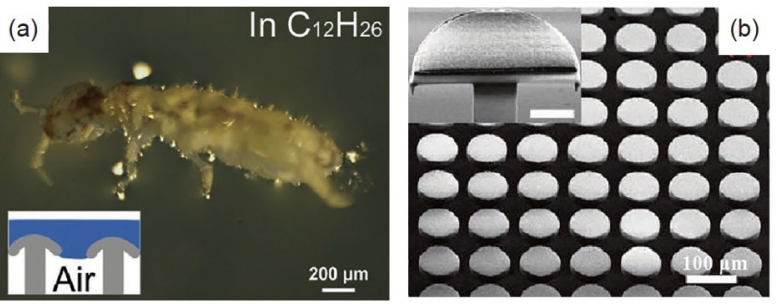

(c)

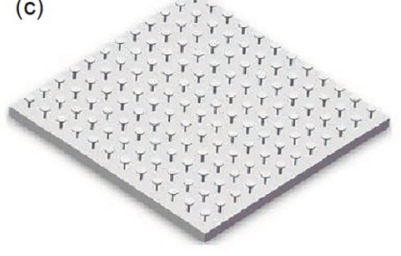

(e)

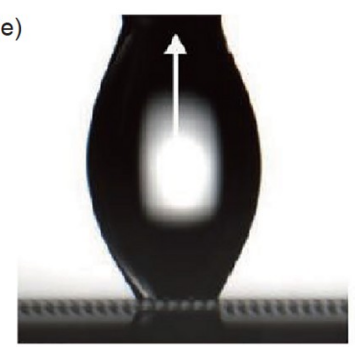

(f)

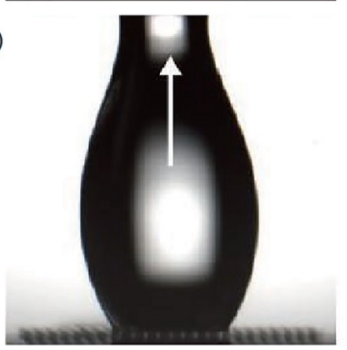

(d)
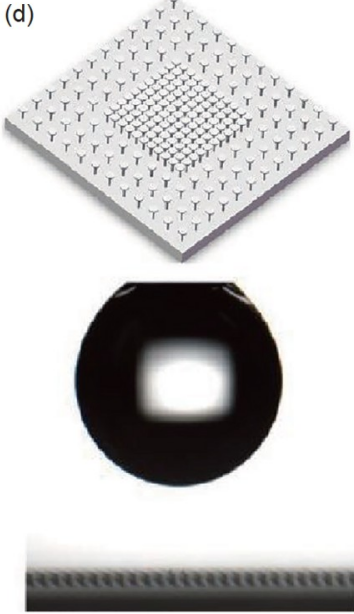

$147^{\circ}$
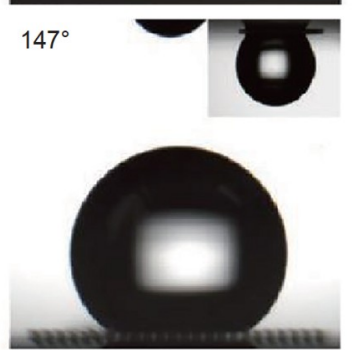

图 8 (a) 跳虫皮肤的浸润效果图 ${ }^{[46]}$; (b) 制备的双重凹腔结 构阵列电镜表征图; (c) 具有疏油能力的均匀阵列示意图; (d) 具有液滴捕获能力的图案化阵列示意图; (e) 疏油效果图; (f) 液滴捕获效果图 ${ }^{[47]}$ (网络版彩图)

Figure 8 (a) Images of Folsomia candida in $n$-dodecane [46]; (b) SEM images of the micro doubly re-entrant arrays; (c) Scheme of the doubly re-entrant array with high contact angle; (d) Scheme of the doubly re-entrant array with liquid adhesion; (e) Digital images demonstrate the anti-adhesion behavior of silicone oil on the corresponding array; (f) Digital images demonstrate the adhesion of silicone oil on the corresponding array [47] (color online).

程操作或自主运行能力的微型机器人成为生物医学工 程领域研究的热点. 而微型机器人的关键在响应执行 单元的构建. 传统构建方案通过在机器人结构中引入 具有电响应、磁响应、热响应材料 ${ }^{[4,50]}$. 而具有可控 形变能力的微结构可控材料则为微型机器人提供了一 种新型操控方案，不仅可以赋予普通材料特殊的响应 形变, 还能够增加响应性材料的灵活度.

德国卡尔斯鲁厄理工学院Wegener团队 ${ }^{[51]}$ 以手性 结构为基本单元开发了具有应力扭转功能的周期性结 构. 如图9所示, 上半部分由左旋结构构成, 下半部分由 右旋结构构成. 当作用力沿坚直方向作用时, 整体结构 可以在水平方向内发生顺时针扭曲. 这一方案发挥了 双光子聚合技术在复杂结构成型方面的优势, 降低了 微执行器中多材料加工的难度.

此外, 相关研究团队将微结构设计与响应性材料 结合实现协同增效, 进一步增加微执行单元的灵活度. Duan等人 ${ }^{[52,53]}$ 在双光子聚合加工水凝胶材料的基础 上，利用微结构网络的组合控制不同响应单元的空间 分布，最终可以演示了毫米尺度“汽车人”从“车”到 “人”的形状改变. 本团队 ${ }^{[54]}$ 在双光子聚合加工中引入 非对称聚合度, 使得加工的微结构能够在液体毛细力 作用下发生定向弯曲. 将制备的结构单元集成到具有 疏水性能的凹腔阵列中, 通过可逆形变起到疏水与亲 水界面之间的切换.

\section{4 总结与展望}

随着更加精细、更加复杂的人工结构被设计与制 造, 微结构可控材料的力学、表界面、应激响应性能 被发现, 并在生物医学领域寻找到合适的应用. 本文 对已有研究中的加工工艺和应用案例进行综述, 目的 在于形成标准化工艺流程, 推动人体器官芯片这一变 革性技术的发展.

人体器官芯片是一种可用于微缩人工组织和器官 培养的微流控芯片. 通过构建与人体高度类似的体外 环境进行细胞培养, 可以帮助了解人体器官的工作机

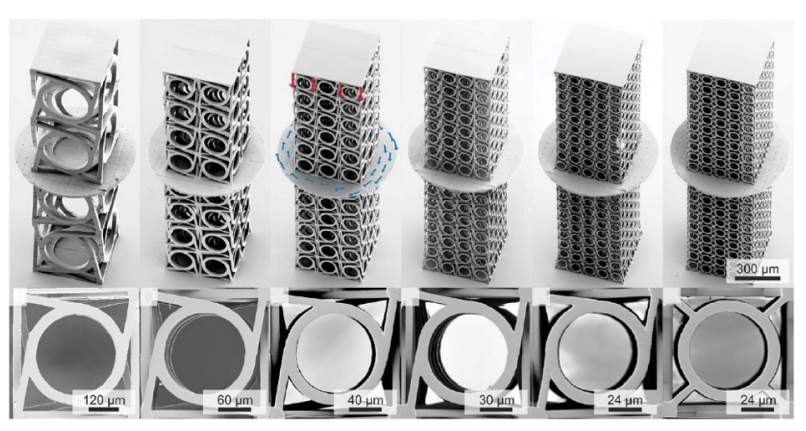

图 9 不同几何参数的基本单元级整体结构的应力形变效 果图 ${ }^{[51]}$ (网络版彩图)

Figure 9 Images of the unit cells within different geometric parameters and the corresponding twist under the axial strain [51] (color online). 
制及器官之间的相互作用, 为药物笁选和病理模型提 供高仿真研究和测试平台. 作为替代动物实验、降低 药物研发成本的变革性工具, 人体器官芯片正在成为 生物医学领域的“卡脖子”关键技术. 经过十年的发展, 人体器官芯片经历了从简单的微流体通道结构到集成 有细胞外支架、仿生血管、原位传感单元等多部件架 构的复杂系统. 具有可控力学、浸润及形变能力的微 结构可控材料能够与微流体芯片集成，进一步简化芯 片内功能器件的加工难度, 提高人工微器官和相关数 据的可靠性(图10).

随着人体器官芯片研究的深入，微结构可控材料 还需要继续优化加工工艺和结构设计：

（1）高精度、高效率的加工方案. 对于微结构可控 材料而言，双光子聚合在带来亚微米级加工精度的同 时，也造成了加工范围小，加工效率低的困扰. 虽然已 有研究在一定程度上解决了部分问题，但是与双光子 聚合加工配套的精密机械位移装置的工作行程局限在 几百微米级别, 面对毫米以上级的加工目标往往需要 低精度的大行程位移台实施拼接操作。这一过程中引 入的拼接误差对于整体性能造成巨大损伤。同时，逐 点扫描的加工模式决定了材料不是一次性成型，这不 仅影响了加工效率，还容易破坏结构质量. 因此，解决 双光子聚合技术中高精度与大行程、高效率之间矛盾 是未来提升微结构可控材料性能的关键，也是推动微 结构可控材料从实验室走向实际应用的基础.
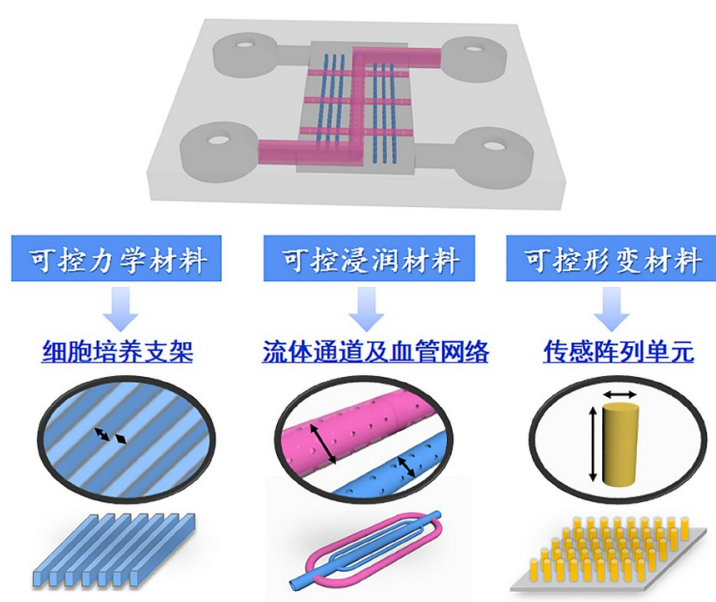

图 10 微结构可控材料在人体器官芯片中的应用示意图 (网络版彩图)

Figure 10 Scheme of the controlled microstructural architectures for organ-on-a-chip (color online).

(2) 多重性能的融合运用. 现有研究中仅研究了材 料在机械、表界面、应激形变等方面单一性能.而实 际应用中，需要考虑材料多种性能的匹配．例如微型 机器人的研究中，除了要考虑响应单元的灵敏度，也 要考虑特定应用场景下如何维持其机械稳定性、以及 生理环境下对于周围液体的排斥与吸附. 因此, 在后续 研究中要根据不同应用场景下的实际需求，组合不同 性能的基本结构，实现结构与性能的多重融合，确立 更加实用的微结构可控材料模型.

\section{参考文献}

1 Ding H, Zhang Q, Gu H, Liu X, Sun L, Gu M, Gu Z. Adv Funct Mater, 2021, 30: 1901760

2 Boatti E, Vasios N, Bertoldi K. Adv Mater, 2017, 29: 1700360

3 MacGregor-Ramiasa MN, Vasilev K. Adv Mater Interfaces, 2017, 4: 1700381

4 Schaedler TA, Jacobsen AJ, Torrents A, Sorensen AE, Lian J, Greer JR, Valdevit L, Carter WB. Science, 2011, 334: 962-965

5 Truby RL, Lewis JA. Nature, 2016, 540: 371-378

6 Zhou LY, Fu J, He Y. Adv Funct Mater, 2020, 30: 2000187

7 LaFratta CN, Fourkas JT, Baldacchini T, Farrer RA. Angew Chem Int Ed, 2007, 46: 6238-6258

8 Li L, Fourkas JT. Mater Today, 2007, 10: 30-37

9 Shoji S, Kawata S. Appl Phys Lett, 1999, 75: 737-739

10 Zheng X, Lee H, Weisgraber TH, Shusteff M, DeOtte J, Duoss EB, Kuntz JD, Biener MM, Ge Q, Jackson JA, Kucheyev SO, Fang NX, Spadaccini CM. Science, 2014, 344: 1373-1377

11 Sun C, Fang N, Wu DM, Zhang X. Sens Actuat A-Phys, 2005, 121: 113-120

12 Tumbleston JR, Shirvanyants D, Ermoshkin N, Janusziewicz R, Johnson AR, Kelly D, Chen K, Pinschmidt R, Rolland JP, Ermoshkin A, Samulski ET, DeSimone JM. Science, 2015, 347: 1349-1352

Walker DA, Hedrick JL, Mirkin CA. Science, 2019, 366: 360-364 
Regehly M, Garmshausen Y, Reuter M, König NF, Israel E, Kelly DP, Chou CY, Koch K, Asfari B, Hecht S. Nature, 2020, 588: 620-624

Anscombe N. Nat Photon, 2010, 4: 22-23

Bauer J, Hengsbach S, Tesari I, Schwaiger R, Kraft O. Proc Natl Acad Sci USA, 2014, 111: 2453-2458

Jang D, Meza LR, Greer F, Greer JR. Nat Mater, 2013, 12: 893-898

Li L, Gattass RR, Gershgoren E, Hwang H, Fourkas JT. Science, 2009, 324: 910-913

Frenzel T, Findeisen C, Kadic M, Gumbsch P, Wegener M. Adv Mater, 2016, 28: 5865-5870

Gan Z, Turner MD, Gu M. Sci Adv, 2016, 2: e1600084

Gan Z, Cao Y, Evans RA, Gu M. Nat Commun, 2013, 4: 2061

Kato J, Takeyasu N, Adachi Y, Sun HB, Kawata S. Appl Phys Lett, 2005, 86: 044102

Li X, Cao Y, Tian N, Fu L, Gu M. Optica, 2015, 2: 567

Saha SK, Wang D, Nguyen VH, Chang Y, Oakdale JS, Chen SC. Science, 2019, 366: 105-109

Yang L, Chen X, Wang L, Hu Z, Xin C, Hippler M, Zhu W, Hu Y, Li J, Wang Y, Zhang L, Wu D, Chu J. Adv Funct Mater, 2019, $29: 1905745$

Wang C, Yang L, Hu Y, Rao S, Wang Y, Pan D, Ji S, Zhang C, Su Y, Zhu W, Li J, Wu D, Chu J. ACS Nano, 2019, 13: 4667-4676

Lee SW, Jafary-Zadeh M, Chen DZ, Zhang YW, Greer JR. Nano Lett, 2015, 15: 5673-5681

Xia X, Afshar A, Yang H, Portela CM, Kochmann DM, Di Leo CV, Greer JR. Nature, 2019, 573: 205-213

Meza LR, Das S, Greer JR. Science, 2014, 345: 1322-1326

Zheng X, Smith W, Jackson J, Moran B, Cui H, Chen D, Ye J, Fang N, Rodriguez N, Weisgraber T, Spadaccini CM. Nat Mater, 2016, 15: 11001106

Cao YY, Takeyasu N, Tanaka T, Duan XM, Kawata S. Small, 2009, 5: 1144-1148

Cao Y, Gu M. Appl Phys Lett, 2013, 103: 213104

Tabrizi S, Cao Y, Cumming BP, Jia B, Gu M. Adv Opt Mater, 2016, 4: 529-533

Blasco E, Müller J, Müller P, Trouillet V, Schön M, Scherer T, Barner-Kowollik C, Wegener M. Adv Mater, 2016, 28: 3592-3595

Eckel ZC, Zhou C, Martin JH, Jacobsen AJ, Carter WB, Schaedler TA. Science, 2016, 351: 58-62

Bauer J, Schroer A, Schwaiger R, Kraft O. Nat Mater, 2016, 15: 438-443

Torrents A, Schaedler TA, Jacobsen AJ, Carter WB, Valdevit L. Acta Mater, 2012, 60: 3511-3523

Schaedler TA, Jacobsen AJ, Carter WB. Science, 2013, 341: 1181-1182

Rys J, Valdevit L, Schaedler TA, Jacobsen AJ, Carter WB, Greer JR. Adv Eng Mater, 2014, 16: 889-896

Meza LR, Zelhofer AJ, Clarke N, Mateos AJ, Kochmann DM, Greer JR. Proc Natl Acad Sci USA, 2015, 112: 11502-11507

Liontas R, Greer JR. Acta Mater, 2017, 133: 393-407

Hollister SJ. Nat Mater, 2005, 4: 518-524

Yang B, Wolfenson H, Chung VY, Nakazawa N, Liu S, Hu J, Huang RYJ, Sheetz MP. Nat Mater, 2019, 19: 239-250

Guell Izard A, Bauer J, Crook C, Turlo V, Valdevit L. Small, 2019, 15: 1903834

Sun L, Gu H, Liu X, Ni H, Li Q, Zeng Y, Chang N, Zhang D, Chen H, Li Z, Zhao X, Gu Z. Nat Commun, 2020, 11: 5732

Liu X, Gu H, Wang M, Du X, Gao B, Elbaz A, Sun L, Liao J, Xiao P, Gu Z. Adv Mater, 2018, 30: 1800103

Liu X, Gu H, Ding H, Du X, He Z, Sun L, Liao J, Xiao P, Gu Z. Small, 2019, 15: 1902360

Liu X, Gu H, Ding H, Du X, Wei M, Chen Q, Gu Z. Adv Sci, 2020, 7: 2000878

$\mathrm{Hu}$ W, Lum GZ, Mastrangeli M, Sitti M. Nature, 2018, 554: 81-85

Miskin MZ, Cortese AJ, Dorsey K, Esposito EP, Reynolds MF, Liu Q, Cao M, Muller DA, McEuen PL, Cohen I. Nature, 2020, 584: 557-561

Frenzel T, Kadic M, Wegener M. Science, 2017, 358: 1072-1074

Jin D, Chen Q, Huang TY, Huang J, Zhang L, Duan H. Mater Today, 2020, 32: 19-25

Huang TY, Huang HW, Jin DD, Chen QY, Huang JY, Zhang L, Duan HL. Sci Adv, 2020, 6: eaav8219

Liu X, Wei M, Wang Q, Tian Y, Han J, Gu H, Ding H, Chen Q, Zhou K, Gu Z. Adv Mater, 2021, 33: 2100332 


\title{
Fabrication of controlled microstructural architectures and applica- tion in biomedical engineering
}

\author{
Haibo Ding, Keliang Liu, Mengxiao Wei, Yijun Guo, Hongcheng Gu, Zhongze Gu* \\ State Key Laboratory of Bioelectronics, School of Biological Science and Medical Engineering, Southeast University, Nanjing 210096, China \\ *Corresponding author (email: gu@seu.edu.cn)
}

\begin{abstract}
The artificially designed microstructures and nanostructures with controlled properties could be defined as controlled microstructural architectures (CMAs). The properties of the CMAs arise from the feature size, the geometrical configuration, and the patterns of the specially designed structures rather than the physical or chemical properties of the base materials. The key issue of the CMAs is to realize the architectures with growing structural complexity and multilevel hierarchy. In this review, we focus on the recent progress of the fabrication methods for CMAs. The multi-scale additive manufacturing techniques for polymers and metal coating were highlighted to increase the whole performance. Besides, the biomedical applications of the CMAs were summarized in low-density materials, droplet manipulation, and microrobots. This review also covers some remaining challenges for CMAs, along with future outlooks.
\end{abstract}

Keywords: controlled microstructural architectures, multi-scale, additive manufacturing, biomedical engineering

doi: $10.1360 /$ SSC-2021-0073 